\title{
The ultra-cool white dwarf companion of PSR J0751+1807
}

\author{
C. G. Bassa ${ }^{1}$, M. H. van Kerkwijk ${ }^{2}$, and S. R. Kulkarni ${ }^{3}$ \\ 1 Astronomical Institute, Utrecht University, PO Box 80 000, 3508 TA Utrecht, The Netherlands \\ e-mail: c.g.bassa@astro.uu.nl \\ 2 Department of Astronomy and Astrophysics, University of Toronto, 60 Saint George Street, Toronto, ON M5S 3H8, Canada \\ 3 Palomar Observatory, California Institute of Technology 105-24, Pasadena, CA 91125, USA
}

Received 7 October 2005 / Accepted 29 December 2005

\section{ABSTRACT}

We present optical and near-infrared observations with Keck of the binary millisecond pulsar PSR J0751+1807. We detect a faint, red object with $R=25.08 \pm 0.07, B-R=2.5 \pm 0.3$, and $R-I=0.90 \pm 0.10-$ at the celestial position of the pulsar and argue that it is the white dwarf companion of the pulsar. The colours are the reddest among all known white dwarfs, and indicate a very low temperature, $T_{\text {eff }} \approx 4000 \mathrm{~K}$. This implies that the white dwarf cannot have the relatively thick hydrogen envelope that is expected on evolutionary grounds. Our observations pose two puzzles. First, while the atmosphere was expected to be pure hydrogen, the colours are inconsistent with this composition. Second, given the low temperature, irradiation by the pulsar should be important, but we see no evidence for it. We discuss possible solutions to these puzzles.

Key words. stars: pulsars: individual: PSR J0751+1807 - stars: binaries: close - stars: neutron - stars: white dwarfs

\section{Introduction}

Among the pulsars in binaries, the largest group, the low-mass binary pulsars, has low-mass white dwarf companions. Before the companions became white dwarfs, their progenitors filled their Roche lobe and mass was transferred to the neutron stars, thereby spinning them up and decreasing their magnetic fields. Considerations of the end of this stage, where the white dwarf progenitor's envelope becomes too tenuous to be supported further, allow one to make predictions for relations between the orbital period and white dwarf mass, and orbital period and eccentricity (for a review, e.g., Phinney \& Kulkarni 1994; Stairs 2004). Furthermore, after the cessation of mass transfer, two clocks will start ticking at the same time: the neutron star, now visible as a millisecond pulsar, will spin down, while the secondary will contract to a white dwarf and start to cool. Consequently, the spin-down age of the pulsar should equal the cooling age of the white dwarf.

From optical observations of white dwarf companions to millisecond pulsars one can estimate the white dwarf cooling age and compare it with the pulsar spin-down age. Initial attempts to do this (Hansen \& Phinney 1998a,b; Schönberner et al. 2000) revealed a dichotomy in the cooling properties of white dwarfs in the sense that some white dwarf companions to older pulsars have cooled less than those of younger pulsars. In particular, the companions of PSR J0437-4715 (Danziger et al. 1993; van Straten et al. 2001) and PSR B1855+09 (van Kerkwijk et al. 2000; Ryba \& Taylor 1991) have temperatures of about 4000-5000 K, with characteristic pulsar ages of 5 Gyr. This is in contrast to the companion of PSR J1012+5307 (Lorimer et al. 1995; van Kerkwijk et al. 1996; Callanan et al. 1998), which has a higher temperature $(8600 \mathrm{~K})$, while it orbits an older pulsar $(8.9 \mathrm{Gyr})$.

A likely cause for this dichotomy is the difference in the thickness of the envelope of hydrogen surrounding the helium core of the white dwarf (Alberts et al. 1996). After the cessation of mass transfer, the white dwarfs have relatively thick $\left(\sim 10^{-2} M_{\odot}\right)$ hydrogen envelopes which are able to sustain residual hydrogen shell-burning, keeping the white dwarf hot and thereby slowing the cooling (Driebe et al. 1998). The shell burning, however, can become unstable and lead to thermal flashes which can reduce the mass of the envelope. White dwarfs with such reduced, relatively thin $\left(\$ 10^{-3} M_{\odot}\right)$ hydrogen envelopes cannot burn hydrogen and, as a result, cool faster. The transition between thick and thin hydrogen envelopes was predicted to lie near $0.18-0.20 M_{\odot}$ (where heavier white dwarfs have thin envelopes; Alberts et al. 1996; Sarna et al. 2000; Althaus et al. 2001).

Until recently, PSR J1012+5307, with an orbital period $P_{\mathrm{b}}=0.60 \mathrm{~d}$, was the only system for which a thick hydrogen envelope was required to match the two timescales. Given the relation between the white dwarf mass and the orbital period (Joss et al. 1987; Rappaport et al. 1995; Tauris \& Savonije 1999), companions in similar or closer orbits should have similar or lower mass, and thus have thick hydrogen envelopes as well. This was confirmed by the recent discovery of two new, 
nearby, binary millisecond pulsars with orbital periods similar to that of PSR J1012+5307; PSR J1909-3744 (1.53 d, Jacoby et al. 2005) and PSR J1738+0333 (0.354 d, Jacoby et al., in prep.; see van Kerkwijk et al. 2005 for preliminary results). For both, the temperatures and characteristic ages are similar to those of PSR J1012+5307, and thus one is led to the same need for a thick hydrogen envelope. These discoveries, combined with the thin envelopes inferred for PSR J0034-0534 $(1.59 \mathrm{~d})$ and binaries with longer periods, suggest that the transition occurs at a mass that corresponds to an orbital period just over $1.5 \mathrm{~d}$ (van Kerkwijk et al. 2005). All systems with shorter orbital periods should have thick hydrogen envelopes.

The two known millisecond pulsars with white dwarf companions that have shorter orbital periods than PSR J1012+5307 but do not have optical counterparts, are PSR J0613-0200, with a $1.20 \mathrm{~d}$ period, and PSR J0751+1807, which has the shortest orbital period of all binary millisecond pulsars with $M_{\mathrm{c}}>0.1 M_{\odot}$ companions, $0.26 \mathrm{~d}$ (Lundgren et al. 1995). The latter system is of particular interest because the companion mass has been determined from pulsar timing $\left(M_{\mathrm{WD}}=0.19 \pm\right.$ $0.03 M_{\odot}$ at $95 \%$ confidence; Nice et al. 2005), so that one does not have to rely on the theoretical period-mass relationship. Intriguingly, for PSR J0751+1807, optical observations from Lundgren et al. (1996) set a limit to the temperature of $9000 \mathrm{~K}$, which is only marginally consistent with it having a thick hydrogen envelope. Based on this, Ergma et al. (2001), suggested the hydrogen envelope may have been partially lost due to irradiation by the pulsar.

The faintness of the companion to PSR J0751+1807 aroused our curiosity and motivated us to obtain deep observations to test the theoretical ideas discussed above. We describe our observations in Sect. 2, and use these to determine the temperature, radius and cooling history in Sect. 3. In Sect. 4, we investigate irradiation by the pulsar, finding a surprising lack of evidence for any heating. We discuss our results in Sect. 5.

\section{Observations and data reduction}

The PSR J0751+1807 field was observed with the 10 meter Keck I and II telescopes on Hawaii on five occasions. On December 11, 1996 the Low Resolution Imaging Spectrometer (LRIS, Oke et al. 1995) was used to obtain $B$ and $R$-band images, while the Echellette Spectrograph and Imager (ESI, Sheinis et al. 2002) was used on December 21, 2003 to obtain deeper $B$ and $R$-band, as well as $I$-band images. The $R$-band filter used that night was the non-standard "Ellis $R$ " filter. The observing conditions during the 1996 night were mediocre, with $0 .{ }^{\prime} 8-1$.' 1 seeing and some cirrus appearing at the end of the night. The conditions were photometric during the 2003 night, and the seeing was good, $0 .{ }^{\prime} 6-0 .{ }^{\prime} 8$. The third and fourth visit were with LRIS again, now at Keck I, on January 7 and 8, 2005. The red arm of the detector was used to obtain $R$-band images. The seeing on the first night in 2005 was rather bad, about 1". 5 and improved to about $1 . .0$ on the second night. The conditions on these nights were not photometric. Finally, a series of 36 dithered exposures, each consisting of 5 co-added $10 \mathrm{~s}$ integrations, were taken through the $K_{\mathrm{s}}$ filter with the Near Infrared Camera (NIRC; Matthews \& Soifer 1994) on January 26, 2005.
Table 1. Observation log.

\begin{tabular}{|c|c|c|c|c|}
\hline Field & Time (UT) & Filter & $t_{\text {int }}(\mathrm{s})$ & $\sec z$ \\
\hline \multicolumn{5}{|c|}{ December 11, 1996, LRIS } \\
\hline \multirow[t]{2}{*}{ SA 95} & $08: 23-08: 25$ & $R$ & $2+10$ & 1.07 \\
\hline & $08: 27-08: 29$ & $B$ & $2+10$ & 1.07 \\
\hline \multirow[t]{2}{*}{ SA 95} & 09:28-09:31 & $B$ & $2+10$ & 1.07 \\
\hline & $09: 33-09: 35$ & $R$ & $2+10$ & 1.08 \\
\hline \multirow[t]{4}{*}{ PSR J0751+1807 } & 09:45 & $R$ & 10 & 1.39 \\
\hline & 09:47-09:59 & $R$ & $2 \times 300$ & 1.36 \\
\hline & 10:01 & $R$ & 600 & 1.31 \\
\hline & $10: 13$ & $B$ & 600 & 1.26 \\
\hline \multicolumn{5}{|c|}{ December 21, 2003, ESI } \\
\hline \multirow[t]{3}{*}{ PSR J0751+1807 } & 10:06-10:27 & $R$ & $3 \times 360$ & 1.14 \\
\hline & $10: 29-10: 57$ & $I$ & $6 \times 240$ & 1.08 \\
\hline & $11: 00-11: 33$ & $B$ & $3 \times 600$ & 1.04 \\
\hline \multirow[t]{3}{*}{ NGC 2419} & $11: 40$ & $B$ & $10+30$ & 1.06 \\
\hline & $11: 44$ & $R$ & $10+30$ & 1.06 \\
\hline & $11: 47$ & $I$ & $10+30$ & 1.06 \\
\hline \multicolumn{5}{|c|}{ January 7, 2005, LRIS } \\
\hline PSR J0751+1807 & $11: 54-12: 53$ & $R$ & $5 \times 600$ & 1.05 \\
\hline \multicolumn{5}{|c|}{ January 8, 2005, LRIS } \\
\hline PSR J0751+1807 & $11: 42-12: 51$ & $R$ & $6 \times 600$ & 1.05 \\
\hline \multicolumn{5}{|c|}{ January 26,2005, NIRC } \\
\hline PSR J0751+1807 & 08:06-08:56 & $K_{\mathrm{s}}$ & $36 \times 50$ & 1.07 \\
\hline 2MASS $\operatorname{star}^{a}$ & 08:59 & $K_{\mathrm{s}}$ & 0.4 & 1.02 \\
\hline
\end{tabular}

a 2MASS J07510621+1807253.

The conditions were photometric with 0.' 6 seeing. Standard stars (Landolt 1992; Stetson 2000) were observed in 1996 and 2003, while a 2MASS star (Cutri et al. 2003) in the vicinity of PSR J0751+1807 was observed to calibrate the NIRC data. A $\log$ of the observations is given in Table 1.

The images were reduced using the Munich Image Data Analysis System (MIDAS). The BRI images were biassubtracted and flat-fielded using dome flats. The longer exposures in each filter were aligned using integer pixel offsets, and co-added to create average images. The near-infrared images were corrected for dark current using dark frames with identical exposure times and number of co-adds as those used for the science frames. Next, a flatfield frame was created by median combining the science frames. After division by this flatfield, the science frames were registered using integer pixel offsets and averaged.

\subsection{Astrometry}

For the astrometric calibration, we selected 14 stars from the second version of the USNO CCD Astrograph catalogue (UCAC2; Zacharias et al. 2004) that overlapped with the $10 \mathrm{~s}$ $R$-band LRIS image of December 1996. Of these, 11 were not 
saturated and appeared stellar and unblended. The centroids of these objects were measured and corrected for geometric distortion using the bi-cubic function determined by J. Cohen (1997, priv. comm. $)^{1}$. We fitted for zero-point position, plate scale and position angle. The inferred uncertainty in the singlestar measurement of these 11 stars is 0.057 and $0{ }^{\prime} 083$ in right ascension and declination, respectively, and is consistent with expectations for the UCAC measurements of approximately 0.'020 for stars of 14th magnitude and 0.'070 for stars 2 mag fainter.

This solution was transferred to the $10 \mathrm{~min} R$-band LRIS image using 91 stars that were present on both images and were stellar, unsaturated and not blended. Again the zeropoint position, plate scale and position angle were left free in the fit and the final residuals were 0.'016 and 0.'019 in right ascension and declination. The UCAC is on the International

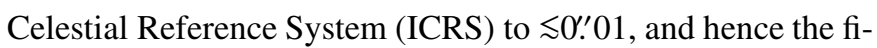
nal systematic uncertainty with which our coordinates are on the ICRS is dominated by our first step, and is $\sim 0$ ' $^{\prime} 03$ in each coordinate.

Our images, with the position of PSR J0751+1807 (Nice et al. 2005) indicated, are shown in Fig. 1. On the 10 min LRIS $R$-band images from 1996 and 2005, we find a faint object, hereafter star X, at the position of the pulsar. It is also, though marginally, present in the two 5 min $R$-band images from 1996, but not detected in the $10 \mathrm{~min} B$-band LRIS image of that observing run. Star X is clearly present in the 2003 ESI $R$ and $I$-band images, and marginally in the $B$-band image. It is not detected in the near-infrared observations (Fig. 1).

Positions for star $\mathrm{X}$ and other objects inferred using the astrometry of the 10 min LRIS $R$-band image are listed in Table 2. The pulsar position at the time of the 1996 LRIS observation, using the Nice et al. (2005) position and proper motion, is $\alpha_{\mathrm{J} 2000}=07^{\mathrm{h}} 51^{\mathrm{m}} 09^{\mathrm{s}} .1574(1), \delta_{\mathrm{J} 2000}=+18^{\circ} 07^{\prime} 38^{\prime \prime} .624(10)$. We find that star $\mathrm{X}$ is offset from the pulsar position by $-0 .{ }^{\prime} 01 \pm$ $0{ }^{\prime} 06$ in right ascension and 0 . $^{\prime} 04 \pm 0 .{ }^{\prime} 06$ in declination, well within the $1 \sigma$ uncertainties (including those on the pulsar position). Given the low density of about 47 stars per square arcminute and the excellent astrometry, the probability of a chance coincidence in the $95 \%$ confidence error circle, which has a radius of $0 ! 24$, is only $0.1-0.2 \%$. Since, as we will see, it is hard to envisage how the companion could be fainter than the object detected, we are confident that star $\mathrm{X}$ is the companion of PSR J0751+1807.

\subsection{Photometry}

The DAOPHOT II package (Stetson 1987), running inside MIDAS, was used for the photometry on the averaged images. We followed the recommendations of Stetson (1987): instrumental magnitudes were obtained through point spread function (PSF) fitting and aperture photometry on brighter stars was used to determine aperture corrections.

For the calibration of the optical images, instrumental magnitudes of the standard stars, determined using aperture

\footnotetext{
1 http://alamoana.keck.hawaii.edu/inst/lris/ coordinates.html
}
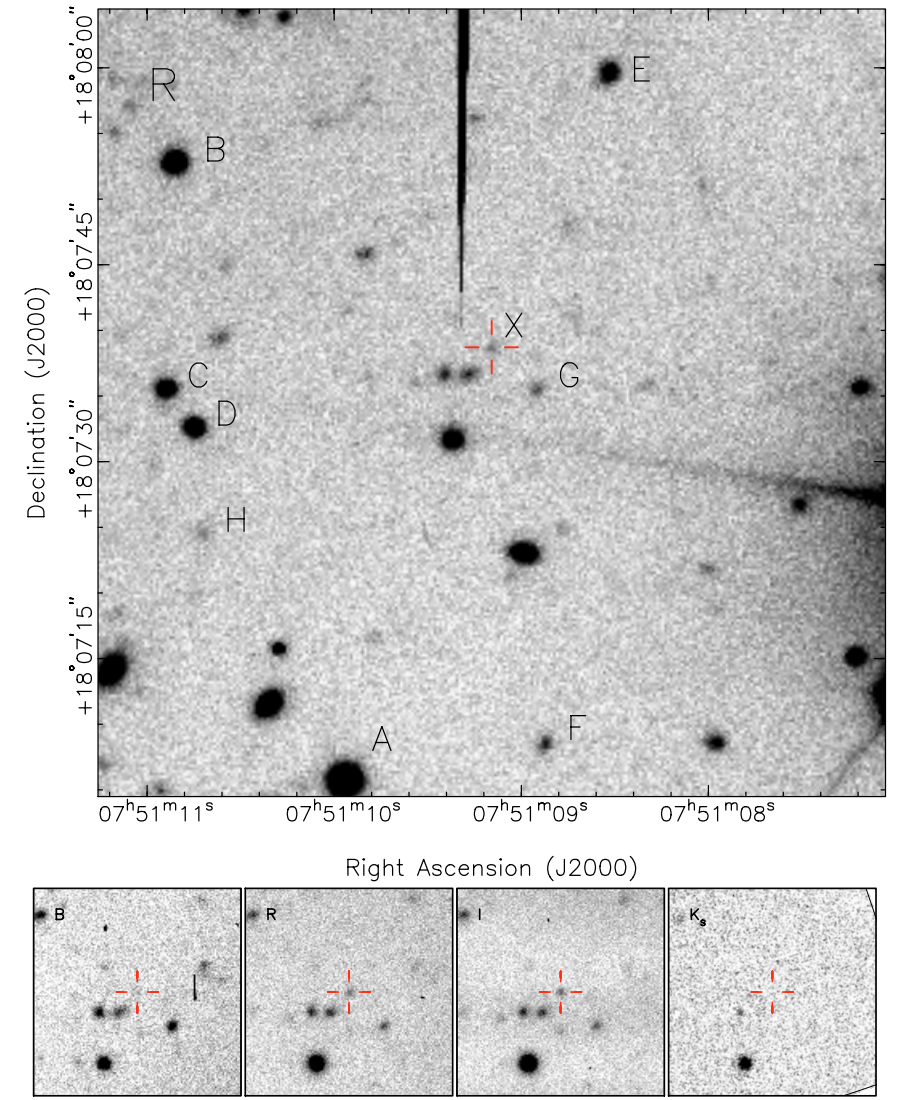

Fig. 1. Images of the field of PSR J0751+1807. The upper figure shows a $1^{\prime} \times 1^{\prime}$ subsection of the averaged $6 \times 10 \mathrm{~m} R$-band image obtained with LRIS on January 8,2005 . The bottom four figures show $20^{\prime \prime} \times 20^{\prime \prime}$ subsections of the $B, R$ and $I$-band averages observed with ESI in 2003 and the $K_{\mathrm{s}}$-band image observed with NIRC in 2005. The tick marks all have a length of $1^{\prime \prime}$ and are centered on the pulsar timing position.

photometry, were compared against the values of Stetson (2000). We used the standard Keck extinction coefficients of $0.17,0.11$ and $0.07 \mathrm{mag}$ per airmass for $B, R$ and $I$, respectively. Colour terms were not required for the LRIS $B$ and $R$ bands, but were significant for the ESI bands: $0.107(B-R)$ for $B, 0.083(B-R)$ for $R$, and $-0.004(R-I)$ for $I$, i.e., the ESI $B$, $R$ are redder than the standard bands, while ESI $I$ is slightly bluer. The root-mean-square residuals of the ESI calibrations are about 0.05 mag in $B$, and $0.03 \mathrm{mag}$ in $R$ and $I$, while those of the LRIS calibration are $0.08 \mathrm{mag}$ in $B$ and $0.05 \mathrm{mag}$ in $R$; we adopt these as the uncertainty in the zero-points. The nearinfrared observations were calibrated through aperture photometry with 1 '.5 (10 pix) apertures using the 2MASS star, fitting for a zero-point only, as the difference in airmass between the science and calibration images is small. We adopt an uncertainty in the $K_{\mathrm{s}}$ zero-point of $0.1 \mathrm{mag}$.

Calibrated ESI magnitudes for star $\mathrm{X}$ and selected other stars in the field are listed in Table 2. Star X is barely above the detection limit of the ESI $B$-band observations, hence the large error. It is not detected in the LRIS $B$-band and the NIRC $K_{\mathrm{s}}$-band observations, and, scaling from the magnitude of a star with a signal-to-noise ratio of about 10 and 6 , we estimate the $3 \sigma$ detection limits at $B=26.8$ and $K_{\mathrm{s}}=21.3$, respectively. 
Table 2. LRIS Astrometry and ESI photometry of the companion of PSR J0751+1807 and stars in the field. The nomenclature of the stars is according to Fig. 1. The uncertainties listed in parentheses are instrumental, i.e., they do not include the zero-point uncertainty in the astrometric tie (about $0{ }^{\prime} 03$ in each coordinate) or of photometric calibration ( $0.05 \mathrm{mag}$ in $B$ and $0.03 \mathrm{mag}$ in both $R$ and $I$ ).

\begin{tabular}{|c|c|c|c|c|c|c|c|}
\hline \multirow[t]{2}{*}{ ID } & \multicolumn{2}{|r|}{$\alpha_{2000}$} & \multicolumn{2}{|r|}{$\delta_{2000}$} & \multirow[t]{2}{*}{$B$} & \multirow[t]{2}{*}{$R$} & \multirow[t]{2}{*}{$I$} \\
\hline & $\mathrm{h}$ & $\mathrm{m} \quad \mathrm{s}$ & 。 & , I & & & \\
\hline$X$ & 0751 & 109.158(4) & 1807 & $38.66(6)$ & $27.56(25)$ & $25.08(7)$ & $24.18(7)$ \\
\hline A & 0751 & 109.933(1) & 1807 & $05.97(1)$ & $21.73(1)$ & $19.30(1)$ & 18.31(1) \\
\hline B & 0751 & $10.844(1)$ & 1807 & $52.91(1)$ & $22.80(1)$ & $21.03(1)$ & $20.32(1)$ \\
\hline $\mathrm{C}$ & 0751 & $10.891(1)$ & 1807 & $35.69(1)$ & $24.30(2)$ & 21.81(1) & $20.63(1)$ \\
\hline $\mathrm{D}$ & 0751 & $10.739(1)$ & 1807 & $32.79(1)$ & $24.28(6)$ & $22.50(5)$ & $21.99(6)$ \\
\hline $\mathrm{E}$ & 075 & $08.519(1)$ & 1807 & $59.89(2)$ & $24.56(7)$ & $22.87(5)$ & $22.38(8)$ \\
\hline $\mathrm{F}$ & 0751 & $08.859(2)$ & 1807 & $08.83(3)$ & 24.94(4) & $24.00(5)$ & $23.29(4)$ \\
\hline $\mathrm{G}$ & 0751 & $08.908(4)$ & 1807 & $35.71(5)$ & $25.65(8)$ & $24.51(5)$ & $23.85(6)$ \\
\hline $\mathrm{H}$ & 0751 & $10.691(3)$ & 1807 & $24.69(6)$ & $25.69(7)$ & $24.94(9)$ & $24.34(8)$ \\
\hline
\end{tabular}

The former is consistent with the ESI detection. None of the stars in Table 2 are covered by the small $38^{\prime \prime} \times 38^{\prime \prime}$ field-ofview of NIRC, hence we do not have near-infrared magnitudes for these.

The 1996 LRIS $R$-band magnitude is $25.13 \pm 0.11$, which is consistent with the ESI measurement. Since the conditions during the 1996 LRIS observations may not have been photometric, however, this may be a coincidence. To check for variability, we tied the instrumental LRIS $R$ band magnitudes directly to the ESI $R$ and $I$ ones, using 38 stars that both images had in common and that had magnitude uncertainties below $0.1 \mathrm{mag}$. As expected given the non-standard "Ellis $R$ " filter on ESI, we required a large colour term, $-0.302\left(R_{\text {inst }}-I_{\text {inst }}\right)$, but with this the fit was adequate, with root-mean-square residuals of $0.14 \mathrm{mag}$. Compared to the fit, the ESI minus LRIS difference in $R$-band magnitude is insignificant, $-0.03 \pm$ 0.13 mag. Similarly, comparing instrumental $R$-band magnitudes from 2005 January 7 with those taken 2005 January 8 and 1996 December 11, fitting for an offset only, results in magnitude differences of $0.03 \pm 0.07$ and $-0.16 \pm 0.12$ mag, respectively. Thus, no large variations in brightness are seen; we will see in Sect. 4 that this is somewhat surprising.

\section{Temperature, radius, and cooling history}

We use our observations of star $\mathrm{X}$, the companion of PSR J0751+1807, to constrain its temperature, radius, and atmospheric constituents, and discuss our result that the white dwarf does not have the expected thick hydrogen envelope.

\subsection{Colours, temperature, and atmospheric composition}

We first use the colours of star X to constrain its temperature. The red colours are largely intrinsic, as the maximum reddening towards PSR J0751+1807 $(l=202.73, b=21.09)$ is small,
$E_{B-V}=0.05 \pm 0.01$ (Schlegel et al. 1998). This value is consistent with the low value found for the interstellar absorption $N_{\mathrm{H}} \sim 4 \times 10^{20} \mathrm{~cm}^{-2}$, as estimated from ROSAT X-ray observations of PSR J0751+1807 by Becker et al. (1996). For comparison, the relation by Predehl \& Schmitt (1995) predicts an $N_{\mathrm{H}} \approx 3 \times 10^{20} \mathrm{~cm}^{-2}$ for the above reddening. Given the distance of $\sim 0.6 \mathrm{kpc}$ (Nice et al. 2005), we expect most of the reddening to be in the foreground to the pulsar. Hence, the dereddened colours are $(B-R)_{0}=2.40 \pm 0.27$ and $(R-I)_{0}=$ $0.86 \pm 0.10$.

In Fig. 2a, we compare the intrinsic colours of star $\mathrm{X}$ with those of other white dwarf companions of millisecond pulsars, other white dwarfs, and models. We find that the colours of star X are the reddest for any known millisecond pulsar companion or white dwarf. The pulsar companion that comes closest is that of PSR J0437-4715 $(B-R=2.12 \pm 0.06, R-I=$ $0.56 \pm 0.02$ (Danziger et al. 1993) and negligible extinction $\left.{ }^{2}\right)$; the most similar white dwarf is WD $0346+246(B-R=2.2 \pm$ $0.1, R-I=0.76 \pm 0.08$, Oppenheimer et al. 2001). Thus, star X is likely as cool or even cooler than the $T_{\text {eff }} \simeq 3700 \mathrm{~K}$ inferred for those two sources (PSR J0437-4715: Danziger et al. 1993; Hansen 2002, priv. comm.; WD 0346+246: Oppenheimer et al. 2001; Bergeron 2001).

Also shown in Fig. 2a are colours expected from model atmospheres of Serenelli et al. (2001) and of Hansen (2004, priv. comm.), which are specifically tailored to the low-mass, helium-core companions of millisecond pulsars, as well as those for updated low-gravity $(\log g=7)$, pure hydrogen atmosphere models ${ }^{3}$ of Bergeron et al. (1995). One sees that the colours of the companion of PSR J0437-4715, as well as those of the hotter companions of PSR J1012+5307 and J0218+4232, are consistent with these models. For star X, however, the colours are not consistent, as the models never venture redwards of $R-I \approx 0.7$ and $B-R \approx 2.0$.

The change in direction of the tracks is seen in all models for hydrogen-rich, metal-free atmospheres; it reflects a change in the dominant source of opacity, from bound-free absorption of $\mathrm{H}^{-}$at higher temperatures to collision-induced absorption of $\mathrm{H}_{2}$ at lower ones (Lenzuni et al. 1991; Saumon et al. 1994; Hansen 1998). The latter process is highly non-grey, and leads to absorption predominantly longward of the $R$-band. As a result, the $R-I$ colour becomes bluer with decreasing temperatures, while $B-R$ remains roughly constant.

Could star X have a different composition? Due to the high gravity of white dwarfs, metals settle out of the atmosphere. However, some white dwarfs have atmospheres dominated not by hydrogen, but by helium. For the latter, the opacity sources are all fairly grey, and hence the colours continue to redden with decreasing temperatures. Indeed, the colours of star $\mathrm{X}$ are consistent with the predictions of the updated $\log g=7$ pure helium models after Bergeron et al. (1995) at $T_{\text {eff }} \simeq 4200 \mathrm{~K}$ (Fig. 2a).

\footnotetext{
2 As inferred from the dust maps of Schlegel et al. (1998); Danziger et al. (1993) estimate $E_{B-V}=0.07$ from the work of Knude (1979).

${ }^{3}$ For updated versions of the Bergeron et al. (1995) models, see http://www . astro.umontreal.ca/ 'bergeron/CoolingModels/
} 


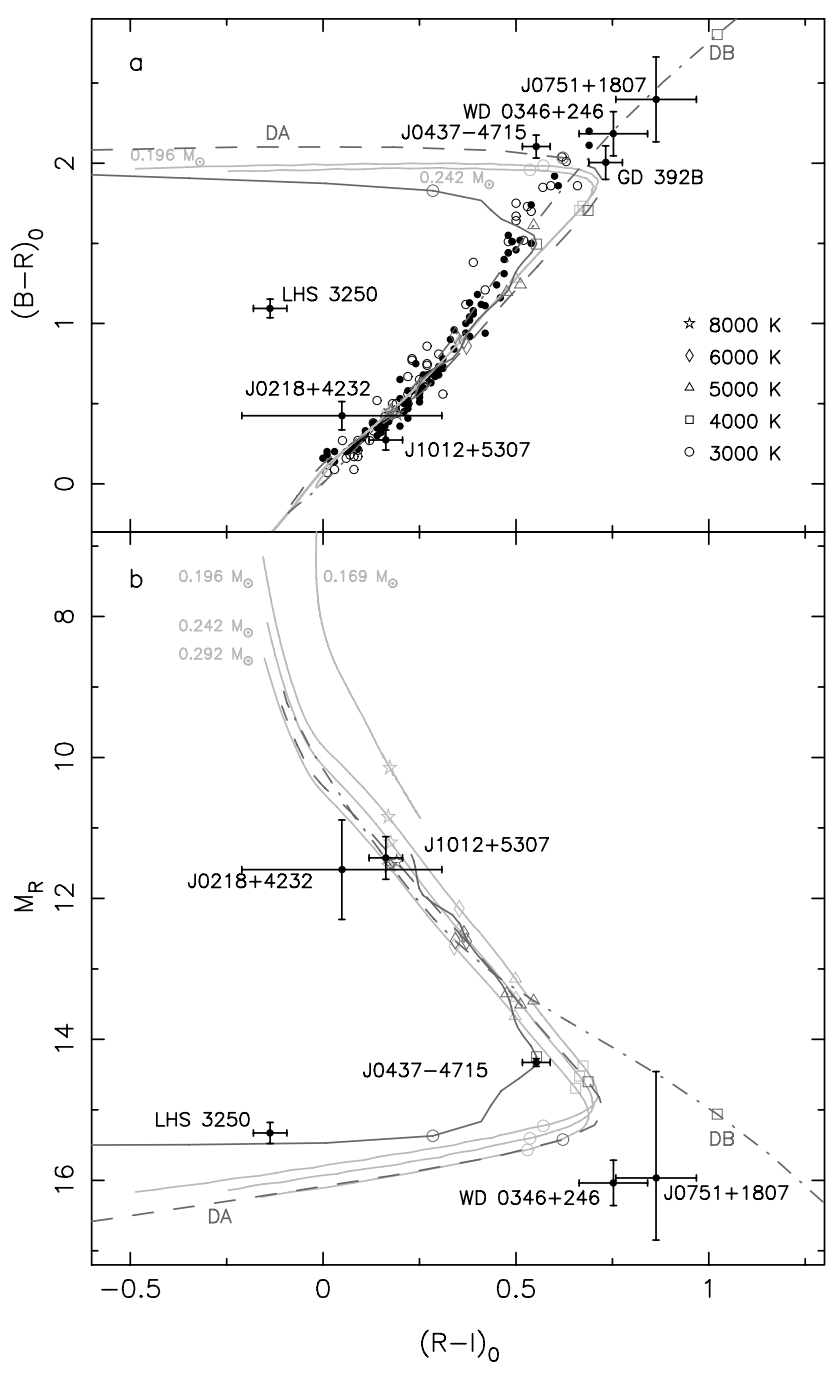

Fig. 2. a) Colour-colour and b) colour-magnitude diagram for the companion of PSR J0751+1807, other millisecond pulsar companions, field white dwarfs, and model predictions. Shown with error bars are PSR J0437-4715 (Danziger et al. 1993), PSR J1012+5307 (Lorimer et al. 1995), PSR J0218+4232 (Bassa et al. 2003) and PSR J0751+1807 (this work), as well as the ultra-cool field white dwarfs LHS 3250 (Harris et al. 1999), WD 0346+246 (Oppenheimer et al. 2001), and GD 392B (Farihi 2004). In the colour-colour diagram, also the full sample of field white dwarfs of Bergeron et al. (2001) is shown, with filled and open circles indicating white dwarfs with and without $\mathrm{H} \alpha$ in their spectrum, respectively. For the colour-magnitude diagram, we used parallax distances where available and distances inferred from the pulsar dispersion measure otherwise; we omitted the Bergeron et al. sample because of the large scatter (even though it likely is largely intrinsic). In both panels, the continuous light grey lines depict helium-core white dwarf cooling models by Serenelli et al. (2001), with masses as indicated. The continuous dark-grey track is for a $0.2 M_{\odot}$ model by B. Hansen (priv. comm.; see description in Hansen \& Phinney 1998a). The dark-grey dashed and dashed-dotted lines are updates of the white dwarf models by Bergeron et al. (1995), for DA (hydrogen rich) and DB (helium rich) composition, respectively, both with $\log g=7$. Temperatures for all models are indicated by different symbols along the track.

From an evolutionary perspective, however, a pure helium atmosphere is not expected. Low-mass white dwarfs such as the companions to millisecond pulsars are all formed from low-mass stars whose evolution was truncated by mass transfer well before helium ignition (for recent models, see Tauris \& Savonije 1999; Nelson et al. 2004). As a result, they should have helium cores surrounded by relatively thick, 0.01 to $1 \%$ of the mass, hydrogen envelopes (Driebe et al. 1998; Althaus et al. 2001). Indeed, among the low-mass white dwarf companions to pulsars (van Kerkwijk et al. 2005) as well as among low-mass white dwarfs in general (Bergeron et al. 2001), only hydrogen-dominated atmospheres have been observed.

In principle, at low temperatures, the hydrogen envelope might become mixed in with the helium core. Even if fully mixed, however, the remaining amounts of hydrogen would strongly influence the spectrum. Indeed, the effects of collisioninduced absorption increase with increasing helium abundance up to $N(\mathrm{He}) / N(\mathrm{H}) \simeq 10^{5}$ (Bergeron \& Leggett 2002).

From Fig. 2a, it is clear that the predictions for hydrogendominated atmospheres are also a somewhat poor match to the colours of the cooler normal white dwarfs with hydrogen in their atmospheres (as inferred from absorption at $\mathrm{H} \alpha$, Bergeron et al. 2001; filled circles in the figure). For most, this appears to be due to missing blue opacity in the models (see Bergeron et al. 2001 for a detailed study); the visual through infrared fluxes are reproduced well by the models, and show unambiguously that collision-induced absorption by $\mathrm{H}_{2}$ is important. Indeed, the absorption is evident in the optical colours of some objects, in particular LHS 3250 (shown in Fig. 2) and SDSS J133739.40+000142.8 (Bergeron \& Leggett 2002, and references therein).

For our purposes, however, the case of the ultra-cool white dwarf WD $0346+246$ is most relevant. For this source, the colours cannot be reproduced with either pure hydrogen or helium, but require a mixed atmosphere, dominated by helium (with fractional hydrogen abundances ranging from $10^{-9}$ to $10^{-1}$, depending on assumptions about the contribution of other opacity sources; Oppenheimer et al. 2001; Bergeron 2001 , though recent work puts these abundances in to doubt, P. Bergeron 2005, priv. comm.). For all cases, the temperature is around $3700 \mathrm{~K}$. The similarity in the colours of WD 0346+246 and star X would suggest that star X has a similar, maybe slightly lower, temperature.

From the above, we find that we cannot determine the temperature of the companion of PSR J0751+1807 with certainty, since we do not know its composition. Most likely, however, it is somewhere between the temperature inferred for WD $0346+246$ and that indicated by the (pure helium) models, i.e. in the range of, say $3500-4300 \mathrm{~K}$.

A more stringent test could be provided by the near-infrared observations, as the $R-K$ colour (which is similar to $R-K_{\mathrm{s}}$ ) differs for different predictions. At a temperature of $4000 \mathrm{~K}$ the $\log g=7$ Bergeron et al. (1995) models predict $R-K$ colours of 2.7 and 1.6 for pure helium and pure hydrogen atmospheres, respectively. For the same temperature, $R-K=1.6$ is predicted by the $0.196 M_{\odot}$ model by Serenelli et al. (2001). Finally, for WD $0346+246$, with presumably a mixed hydrogen/helium atmosphere, Oppenheimer et al. (2001) observed $R-K=-0.7$. Unfortunately, our near-infrared observations only limit the 
colour to $R-K<3.8$, which does not constrain any of these predictions.

\subsection{Brightness, distance and radius}

So far, we have only discussed the colours and temperature. We now turn to the absolute magnitude and radius. In Fig. 2b, we show $M_{R}$ as a function of $R-I$. For star X, we computed the absolute $R$-band magnitude $M_{R}$ using the parallax of $\pi=1.6 \pm 0.8$ mas as measured through radio timing (Nice et al. 2005). The resulting distance of $0.6_{-0.2}^{+0.6} \mathrm{kpc}$ is consistent with that estimated from the dispersion measure which predicts $1.1 \pm 0.2 \mathrm{kpc}$, using a dispersion measure of $30.2489 \pm$ $0.0003 \mathrm{pc} \mathrm{cm}^{-3}$ (Nice et al. 2005) and the recent model of the Galactic electron distribution of Cordes \& Lazio (2002). Correcting for the reddening, this implies $M_{R}=15.97_{-1.51}^{+0.88}$.

Given the similarities in the above absolute magnitude of star X and that of WD $0346+246\left(M_{R}=16.1 \pm 0.3\right.$; Hambly et al. 1999; Oppenheimer et al. 2001), and assuming similar temperature, one finds that the radius of star $\mathrm{X}$ should be comparable to the $R=0.010 R_{\odot}$ for WD $0346+246$ (Bergeron 2001). However, the large uncertainty in the parallax of PSR J0751+1807 allows radii between $0.007-0.021 R_{\odot}$. For the white dwarf mass of $\sim 0.19 M_{\odot}$ inferred from pulse timing (Nice et al. 2005), this is consistent the $\sim 0.022 R_{\odot}$ expected from the $0.196 M_{\odot}$ model by (Serenelli et al. 2001).

As can be seen in Fig. 2, the absolute magnitude is also consistent with the predicted values from the $\log g=7$ pure helium model by Bergeron et al. (1995). At a temperature of $T_{\text {eff }}=4250 \mathrm{~K}$, this model has a radius of $0.020 R_{\odot}$ and a mass of $0.15 M_{\odot}$, somewhat smaller than the observed $0.19 M_{\odot}$. To correct for the small difference in mass, we computed white dwarf radii for the observed temperature and mass of the companion and used these to scale the absolute magnitudes of the pure helium track in Fig. 2. At $0.19 M_{\odot}$ and $T_{\text {eff }}=4000 \mathrm{~K}$, the Panei et al. (2000) helium core white dwarf mass-radius relation predicts $0.021 R_{\odot}$. This is very similar to the radius predicted by the Bergeron et al. (1995) $\log g=7$ pure helium models, and as such, the absolute magnitudes are comparable. We conclude that, with in the large uncertainties on the parallax distance, the absolute magnitude and radius that we derive for the companion of PSR J0751+1807 are consistent with the predictions for a pure helium atmosphere.

We note that of the models presented in Fig. 2, those of Bergeron et al. (1995) have been extensively tested to explain the population of nearby white dwarfs (Bergeron et al. 2001; Bergeron 2001; Bergeron \& Leggett 2002) and use a very detailed description of the white dwarf atmosphere combined with the latest opacities (P. Bergeron 2005, priv. comm.). This is not the case for the models of Serenelli et al. and Hansen, and thus we should be careful in using their models quantitatively. Indeed, as can be seen from Fig. 2, their models do not reproduce the observations of cool white dwarfs well. For instance, for the companion of PSR J0437-4715, which has a well-determined mass of $0.236 \pm 0.017 M_{\odot}$ and distance of $139 \pm 3$ pc (van Straten et al. 2001), the models of Serenelli et al. (2001), while consistent with the observed $B-R$ and $R-I$ well, do not reproduce $R-I$ and $M_{R}$ simultaneously. In contrast, the $0.2 M_{\odot}$ model of Hansen (2004, priv. comm.) does pass through the $R-I, M_{R}$ point, but cannot reproduce both colours. It may be that both problems reflect uncertainties in the model atmospheres used by Hansen and Serenelli et al. (2001). It would be worthwhile to couple the evolutionary models of these authors with the updated, very detailed atmospheric model of Bergeron et al. (1995).

\subsection{Cooling history and nature of the envelope}

Despite the uncertainty in the models and in the composition of the atmosphere, our observations show that the companion of PSR J0751+1807 has cooled much more than expected if the amount of hydrogen was thick enough for significant residual nuclear burning (Sect. 1). Indeed, the temperature is as expected if no residual hydrogen burning occurred. For instance, at the characteristic age of the pulsar, $\tau=7.1 \mathrm{Gyr}$ (Nice et al. 2005), the $0.196 M_{\odot}$ of Serenelli et al. (2001), which has a thin envelope, predicts a temperature of about $3200 \mathrm{~K}$, which is roughly consistent with what is observed. With a pure helium atmosphere, a slightly colder temperature, of $\sim 2500 \mathrm{~K}$, is expected, though this is a less secure estimate due to uncertainties in the opacities (Hansen \& Phinney 1998a).

The presence of a thin (or no) hydrogen envelope is not expected, however, since thick envelopes are inferred for other optically identified companions in short-period systems (see Sect. 1). What could be wrong with this expectation? It was based on two theoretical ideas: (i) that below a certain critical mass, no shell flashes occur and hydrogen layers will be thick; and (ii) that the companion mass monotonously increases with increasing orbital period. These assumptions appeared to be confirmed by the available data: for PSR J0751+1807, with a period of $0.26 \mathrm{~d}$, the companion mass of $0.16-0.21 M_{\odot}$ (95\% conf.; Nice et al. 2005) is similar to what is found for two other short-period systems with companions for which thick hydrogen envelopes are inferred, and less than the masses for longer period systems with thin-envelope companions. Specifically, PSR J1012+5307 (0.60 d, 0.12-0.20 $\left.M_{\odot}\right)$ and PSR J1909-3744 (1.53 d, 0.19-0.22 $M_{\odot}$ ) have thick envelopes while PSR J0437-4715 (5.74 d, 0.20-0.27 $\left.M_{\odot}\right)$ and PSR B1855+09 (12.33 d, 0.24-0.29 $\left.M_{\odot}\right)$ have thin envelopes (see Fig. 3 and van Kerkwijk et al. 2005, and reference therein). Thus, while the uncertainties do not exclude that the companion of PSR J0751+1807 is so massive that it its envelope was diminished by shell flashes, the existing data make it unlikely.

Two explanations for a thin envelope remain. First, there may be differences in metallicity among the progenitors of pulsar companions. Serenelli et al. (2002) studied the evolution of low-mass pulsar companions with sub-solar metallicity and found that, since the thermonuclear flashes are induced by the reactions of the CNO-cycle, the threshold mass between thin and thick hydrogen envelopes increases with decreasing metallicity of the white dwarf progenitor. Thus, it may be that the companion of PSR J0751+1807 had a sufficiently higher metallicity that it was above the threshold for shell flashes, while companions in other short-period systems had lower metallicity 


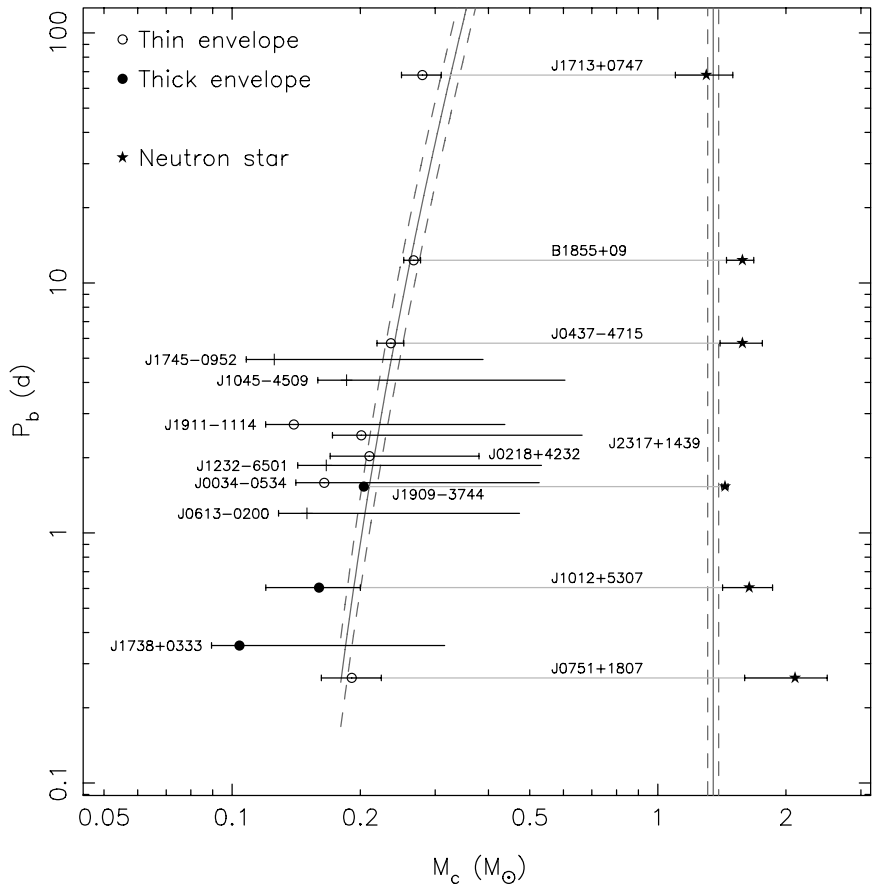

Fig. 3. The orbital period as a function of companion mass for a selection of low-mass binary pulsars outside globular clusters (either with $P_{\mathrm{b}}<5 \mathrm{~d}$ and $M_{\mathrm{WD}, \min }>0.1 M_{\odot}$, or with $P_{\mathrm{b}}<100 \mathrm{~d}$ and a secure companion mass determination). The data is compiled from the ATNF Pulsar Catalogue (Manchester et al. 2005), Stairs (2004) and van Kerkwijk et al. (2005) and references therein. Companion masses are either determined (double error bars, $95 \%$ confidence) or based on the assumption of a $1.35 M_{\odot}$ neutron star and an inclination of $i=90^{\circ}$ (left error bar, minimum mass), $60^{\circ}$ (central symbol, median mass) or $18^{\circ}$ (right side, 5\% probability that $i$ is lower than this value and that the companion is heavier). Different central symbols indicate companions for which a thick or thin hydrogen envelope is inferred from optical measurements. The systems for which the neutron star mass is measured are indicated and connected by a gray line with their companions. The vertical grey lines indicate the $1.35 \pm 0.04 M_{\odot}$ neutron star mass range determined by Thorsett \& Chakrabarty (1999), while the curved grey lines represent the theoretical relation by Tauris \& Savonije (1999) between the white dwarf mass and the orbital period.

and hence were below the threshold, despite having higher masses.

The next possibility is that the white dwarf was indeed formed with a thick envelope, which was subsequently removed by an action other than shell flashes. Based on the upper limit on the temperature of Lundgren et al. (1996), Ergma et al. (2001) already argued that the pulsar companion could not have the thick hydrogen envelope, and they proposed a scenario where part of the envelope was removed by pulsar irradiation. Ergma et al. found that irradiation driven mass-loss could remove as much as $0.01 M_{\odot}$ from the thick hydrogen envelope (mostly while the companion is contracting following the cessation of mass transfer).

A possible problem with the above suggestions, is that none predict the removal of the entire hydrogen envelope, while the observed colours seem most consistent with a pure helium or at least helium-dominated atmosphere.

\section{Irradiation by the pulsar?}

Above, we have treated the companion as if it were an isolated object rather than member of a binary system. Might the presence of a relatively energetic pulsar influence our observations?

The observed pulsar period and period derivative imply a spin-down luminosity $L_{\mathrm{SD}}=(2 \pi)^{2} I \dot{P} / P^{3}=7.5 \times$ $10^{33} I_{45} \mathrm{erg} \mathrm{s}^{-1}$ (Lundgren et al. 1995; Nice et al. 2005), where $I=10^{45} I_{45} \mathrm{~g} \mathrm{~cm}^{2}$ is the pulsar moment of inertia. For a $2.1 M_{\odot}$ pulsar and a $0.19 M_{\odot}$ companion, the orbital separation is $a=2.3 R_{\odot}$, and, consequently, the irradiative flux of the pulsar wind incident on the companion is $f_{\text {irr }}=2.1 \times$ $10^{10} I_{45} \mathrm{erg} \mathrm{s}^{-1} \mathrm{~cm}^{-2}$. This is about twice the flux of the companion itself, $f_{\text {th }}=\sigma T_{\text {eff }}^{4}=1.06 \times 10^{10} \mathrm{erg} \mathrm{s}^{-1} \mathrm{~cm}^{-2}$ for $T_{\text {eff }}=3700 \mathrm{~K}$. Therefore, the presence of the pulsar and its irradiation may be important.

Given the irradiation, one would expect the side of the companion facing the pulsar to be brighter than the side facing away from it. Thus, from Earth, the companion should appear faintest at phase 0.25 and brightest at phase 0.75 (using the convention that at phase 0 , the pulsar is at the ascending node). This is indeed seen in other pulsar binaries, with the black widow pulsar PSR B1957+20 perhaps the most spectacular example (van Paradijs et al. 1988; Fruchter et al. 1988).

For star X, assuming a fraction $\eta$ of the incident flux is absorbed and reradiated as optical flux, the flux from the bright side of the companion should be a factor $1+\frac{2}{3} \eta f_{\text {irr }} / f_{\text {th }}$ brighter (here, the factor $\frac{2}{3}$ reflects projection effects). Observationally, the inferred values of $\eta$ range from 0.1 to 0.6 (Orosz \& van Kerkwijk 2003, and references therein), and thus one expects a maximum change in bolometric flux by a factor 1.13 to 1.8 . For the $R$-band flux, the range is 1.2 to 2.2 (assuming it scales like a black-body spectrum, $\propto T^{6}$ around $3700 \mathrm{~K}$ ). We confirmed this using a detailed light-curve synthesis model (described briefly in Stappers et al. 1999).

For star X, no effect is seen. Using the PSR J0751+1807 ephemeris from Nice et al. (2005), we find that during the ESI $R$-band observations the orbital phase ranged from 0.22 to 0.25 , while the 1996 LRIS $R$-band images were taken at phases 0.86-0.90, and the 2005 LRIS images at phases $0.01-0.14$ on January 7 , and $0.77-0.93$ on January 8. Thus, these observations span the orbital phases necessary to test for any modulation in brightness. Indeed, using the inclination inferred from timing, $i=66_{-7}^{+4} \mathrm{deg}$ (Nice et al. 2005), we find that during the ESI observations only 4 to $5 \%$ of the irradiated part of the companion surface was in view, while during the 1996 LRIS observations is was $78 \%$ to $85 \%$. As a consequence, we expect to see nearly the maximum change in brightness. Nevertheless, in Sect. 2.2, we found no significant variation, $R_{\mathrm{LRIS}}-R_{\mathrm{ESI}}=0.03 \pm 0.13$; thus, to $\sim 99 \%$ confidence, the variation is smaller than $0.3 \mathrm{mag}$, which implies $\eta<0.15$.

The lack of observed modulation could be taken to indicate that the irradiation is not very effective, e.g., because the albedo is large (i.e., $\eta$ is small), the pulsar emission is non-isotropic, or the spin-down luminosity is overestimated. We believe these options are not very likely (for a discussion in a slightly different context, see Orosz \& van Kerkwijk 2003), which leads 
us to consider the only alternative, that one of the assumptions underlying the above estimates is wrong.

In particular, we assumed implicitly that the irradiated flux is reprocessed and re-emitted instantaneously, i.e., transfer of flux inside and around the companion are assumed to have negligible effect. For the companions of black-widow pulsars, this is reasonable, since for these relatively large objects, tides will have ensured synchronous rotation. Any flux transfer would thus have to be due to winds and/or convection, which plausibly happens on a timescale long compared to the thermal time of the layer in which the pulsar flux is reprocessed.

The companion of PSR J0751+1807, however, is well within its Roche-lobe, and tidal dissipation should be negligible. We can estimate its current rotation period from its prior evolution, following the reasoning used by van Kerkwijk \& Kulkarni (1995) for the companion of PSR B0655+64. Briefly, during mass transfer, the companion filled its Roche-lobe and tides ensured the system was synchronised and circularised. Once mass transfer ceased and the companion started to contract to a white dwarf, however, the tides became inefficient, and the rotational evolution of the companion was determined by conservation of angular momentum.

For our estimates, we split the total moment of inertia of the progenitor into two parts, one from the core, $I_{\text {core }}=$ $k_{\text {core }}^{2} M_{\text {core }} R_{\text {core }}^{2}$ and one from the envelope, $I_{\text {env }}=k_{\text {env }}^{2} M_{\text {env }} R_{\mathrm{L}}^{2}$; here $k$ is the radius of gyration and $R_{\mathrm{L}}$ is the radius of the Roche lobe. After contraction of the envelope, one is left with a white dwarf with $I_{\mathrm{WD}}=k_{\mathrm{WD}}^{2} M_{\mathrm{WD}} R_{\mathrm{WD}}^{2}$. If we now assume that $I_{\text {core }} \simeq I_{\mathrm{WD}}$ and ignore differences in radius of gyration, conservation of angular momentum yields $\Omega_{\text {rot }} / \Omega_{\text {orb }} \simeq$ $1+M_{\mathrm{env}} R_{\mathrm{L}}^{2} / M_{\mathrm{WD}} R_{\mathrm{WD}}^{2}$. In reality, the envelope will be more centrally concentrated than the white dwarf, i.e., $k_{\mathrm{env}}<k_{\mathrm{WD}}$, and tidal dissipation will be important in the initial stages of the contraction. This will reduce the spin-up. On the other hand, the hot core of the progenitor will be larger than the white dwarf, i.e., $I_{\text {core }}>I_{\mathrm{WD}}$. In any case, it follows that unless the envelope mass is very small, the white dwarf should be significantly spun up.

Model predictions for the envelope mass of helium-core white dwarfs differ. The $0.196 M_{\odot}$ model by Serenelli et al. (2001), has an envelope mass of $6.7 \times 10^{-3} M_{\odot}$ (as given in Althaus et al. 2001), whereas a model of similar mass $\left(M_{\mathrm{WD}}=\right.$ $0.195 M_{\odot}$ ) by Driebe et al. (1998) has one of $3.1 \times 10^{-2} M_{\odot}$. Using these values, taking $M_{\mathrm{WD}}=0.19 M_{\odot}, R_{\mathrm{WD}}=0.021 R_{\odot}$ and $R_{\mathrm{L}}=0.48 R_{\odot}$, and ignoring differences in $k$, we find current rotation periods a factor $18-85$ faster than the orbital period, or 20 to $5 \mathrm{~min}$. Given that thick envelopes seem inconsistent with the low observed temperature (Sect. 3), the slower end of the range seems more likely.

To estimate the timescale on which the pulsar flux is reprocessed, we assume that the incident particles are predominantly highly energetic, and that they penetrate to, roughly, one Thompson optical depth. This corresponds to a column depth of $N=1.5 \times 10^{24} \mathrm{~cm}^{-2}$, for which the thermal timescale $t \simeq N k T / \sigma T_{\mathrm{eff}}^{4} \simeq 1 \mathrm{~min}$, where the numerical estimate is for $T=T_{\text {eff }}=3700 \mathrm{~K}$. This is shorter than the rotation periods estimated above, suggesting that rotation may not be too important. On the other hand, our estimate is very rough.
For instance, at one Thompson depth, the opacity at optical wavelengths is much smaller than unity for the cool temperatures under consideration (Saumon et al. 1994). Thus, the material likely radiates less efficiently than a black body, which would make the thermal timescale longer. Furthermore, the irradiation will change the temperature and ionisation structure of the atmosphere, further complicating matters. (Indeed, could this be the underlying cause for the fact that the colours deviate so strongly from those expected for a pure hydrogen atmosphere?) Finally, it might induce strong winds which equalise the temperature on both hemispheres (as is the case for Jupiter).

\section{Conclusions}

We have optically identified the white dwarf companion of the binary millisecond pulsar PSR J0751+1807. We find that the companion has the reddest colours of all known millisecond pulsar companions and white dwarfs. These colours indicate that the companion has a very low (ultra-cool) temperature of $T_{\text {eff }} \sim$ 3500-4300 K. Furthermore, the colours suggest that the white dwarf has a pure helium atmosphere, or a helium atmosphere with some hydrogen mixed in, as invoked for the field white dwarf WD 0346+246 which has similar colours (Oppenheimer et al. 2001; Bergeron 2001).

Our observations are inconsistent with evolutionary models, from which one would expect a pure hydrogen atmosphere. Indeed, as for other short-period systems, the hydrogen envelope is expected to be thick enough to sustain significant residual hydrogen burning, leading to temperatures far in excess of those observed. It may be that the mass of the envelope was reduced due to shell flashes or irradiation by the pulsar, as was proposed by Ergma et al. (2001).

However, we see no evidence for irradiation, despite the fact that the pulsar spin-down flux impinging on the white dwarf is roughly twice the observed thermal flux. Clues to what happens might be found from more detailed studies of the spectral energy distribution, or more accurate phase-resolved photometry.

Finally, a deeper observation at infrared wavelengths would allow one to distinguish between the different atmosphere compositions for the companion: for a pure helium atmosphere, black-body like colours are expected, while if any hydrogen is present, the infrared flux would be strongly depressed (as is seen for WD 0346+246). With adaptive optics instruments, such observations should be feasible.

Acknowledgements. We thank Norbert Zacharias for providing preliminary UCAC2 data. We also would like to thank the referee, Pierre Bergeron, for his useful suggestions and for pointing out the existence of his updated models. The observations for this paper were taken at the W. M. Keck Observatory, which is operated by the California Association for Research in Astronomy, a scientific partnership among the California Institute of Technology, the University of California, and the National Aeronautics and Space Administration. It was made possible by the generous financial support of the W. M. Keck Foundation. MIDAS is developed and maintained by the European Southern Observatory. This research made use of the SIMBAD and ADS data bases and of data products from the Two Micron All Sky Survey, which is a joint project of the 
University of Massachusetts and the Infrared Processing and Analysis Center/California Institute of Technology, funded by the National Aeronautics and Space Administration and the National Science Foundation. We acknowledge support from NWO (C.G.B.), NSERC (M.H.v.K.), and from NASA and NSF (S.R.K.).

\section{References}

Alberts, F., Savonije, G. J., van den Heuvel, E. P. J., \& Pols, O. R. 1996, Nature, 380, 676

Althaus, L. G., Serenelli, A. M., \& Benvenuto, O. G. 2001, MNRAS, 324, 617

Bassa, C. G., van Kerkwijk, M. H., \& Kulkarni, S. R. 2003, A\&A, 403, 1067

Becker, W., Trumper, J., Lundgren, S. C., Cordes, J. M., \& Zepka, A. F. 1996, MNRAS, 282, L33

Bergeron, P. 2001, ApJ, 558, 369

Bergeron, P., \& Leggett, S. K. 2002, ApJ, 580, 1070

Bergeron, P., Wesemael, F., \& Beauchamp, A. 1995, PASP, 107, 1047

Bergeron, P., Leggett, S. K., \& Ruiz, M. T. 2001, ApJS, 133, 413

Callanan, P. J., Garnavich, P. M., \& Koester, D. 1998, MNRAS, 298, 207

Cordes, J. M., \& Lazio, T. J. W. 2002 [arXiv:astro-ph/0207156]

Cutri, R. M., Skrutskie, M. F., van Dyk, S., et al. 2003, VizieR Online Data Catalog, 2246

Danziger, I. J., Baade, D., \& della Valle, M. 1993, A\&A, 276, 382

Driebe, T., Schönberner, D., Blöcker, T., \& Herwig, F. 1998, A\&A, 339, 123

Ergma, E., Sarna, M. J., \& Gerškevitš-Antipova, J. 2001, MNRAS, 321,71

Farihi, J. 2004, ApJ, 610, 1013

Fruchter, A. S., Gunn, J. E., Lauer, T. R., \& Dressler, A. 1988, Nature, 334,686

Hambly, N. C., Smartt, S. J., Hodgkin, S. T., et al. 1999, MNRAS, 309, L33

Hansen, B. M. S. 1998, Nature, 394, 860

Hansen, B. M. S., \& Phinney, E. S. 1998a, MNRAS, 294, 557

Hansen, B. M. S., \& Phinney, E. S. 1998b, MNRAS, 294, 569

Harris, H. C., Dahn, C. C., Vrba, F. J., et al. 1999, ApJ, 524, 1000

Jacoby, B. A., Hotan, A., Bailes, M., Ord, S., \& Kulkarni, S. R. 2005, ApJ, 629, L113

Joss, P. C., Rappaport, S., \& Lewis, W. 1987, ApJ, 319, 180

Knude, J. 1979, A\&A, 71, 344

Landolt, A. U. 1992, AJ, 104, 340

Lenzuni, P., Chernoff, D. F., \& Salpeter, E. E. 1991, ApJS, 76, 759

Lorimer, D. R., Lyne, A. G., Festin, L., \& Nicastro, L. 1995, Nature, 376, 393

Lundgren, S. C., Zepka, A. F., \& Cordes, J. M. 1995, ApJ, 453, 419
Lundgren, S. C., Cordes, J. M., Foster, R. S., Wolszczan, A., \& Camilo, F. 1996, ApJ, 458, L33

Manchester, R. N., Hobbs, G. B., Teoh, A., \& Hobbs, M. 2005, AJ, 129,1993

Matthews, K., \& Soifer, B. T. 1994, in Astronomy with Arrays, The Next Generation, ASSL, 190, 239

Nelson, L. A., Dubeau, E., \& MacCannell, K. A. 2004, ApJ, 616, 1124

Nice, D. J., Splaver, E. M., Stairs, I. H., et al. 2005, ApJ, 634, 1242

Oke, J. B., Cohen, J. G., Carr, M., et al. 1995, PASP, 107, 375

Oppenheimer, B. R., Saumon, D., Hodgkin, S. T., et al. 2001, ApJ, 550,448

Orosz, J. A., \& van Kerkwijk, M. H. 2003, A\&A, 397, 237

Panei, J. A., Althaus, L. G., \& Benvenuto, O. G. 2000, A\&A, 353, 970

Phinney, E. S., \& Kulkarni, S. R. 1994, ARA\&A, 32, 591

Predehl, P., \& Schmitt, J. 1995, A\&A, 293, 889

Rappaport, S., Podsiadlowski, P., Joss, P. C., Di Stefano, R., \& Han, Z. 1995, MNRAS, 273, 731

Ryba, M. F., \& Taylor, J. H. 1991, ApJ, 371, 739

Sarna, M. J., Ergma, E., \& Gerškevitš-Antipova, J. 2000, MNRAS, 316,84

Saumon, D., Bergeron, P., Lunine, J. I., Hubbard, W. B., \& Burrows, A. 1994, ApJ, 424, 333

Schönberner, D., Driebe, T., \& Blöcker, T. 2000, A\&A, 356, 929

Schlegel, D. J., Finkbeiner, D. P., \& Davis, M. 1998, ApJ, 500, 525

Serenelli, A. M., Althaus, L. G., Rohrmann, R. D., \& Benvenuto, O. G. 2001, MNRAS, 325, 607

Serenelli, A. M., Althaus, L. G., Rohrmann, R. D., \& Benvenuto, O. G. 2002, MNRAS, 337, 1091

Sheinis, A. I., Bolte, M., Epps, H. W., et al. 2002, PASP, 114, 851

Stairs, I. H. 2004, Science, 304, 547

Stappers, B. W., van Kerkwijk, M. H., Lane, B., \& Kulkarni, S. R. 1999, ApJ, 510, L45

Stetson, P. B. 1987, PASP, 99, 191

Stetson, P. B. 2000, PASP, 112, 925

Tauris, T. M., \& Savonije, G. J. 1999, A\&A, 350, 928

Thorsett, S. E., \& Chakrabarty, D. 1999, ApJ, 512, 288

van Kerkwijk, M. H., \& Kulkarni, S. R. 1995, ApJ, 454, L141

van Kerkwijk, M. H., Bergeron, P., \& Kulkarni, S. R. 1996, ApJ, 467, L89

van Kerkwijk, M. H., Bell, J. F., Kaspi, V. M., \& Kulkarni, S. R. 2000, ApJ, 530, L37

van Kerkwijk, M. H., Bassa, C. G., Jacoby, B. A., \& Jonker, P. G. 2005, in Binary Radio Pulsars, ed. F. A. Rasio, \& I. H. Stairs, ASP Conf. Ser., 328, 357

van Paradijs, J., Allington-Smith, J., Callanan, P., Hassall, B. J. M., \& Charles, P. A. 1988, Nature, 334, 684

van Straten, W., Bailes, M., Britton, M., et al. 2001, Nature, 412, 158

Zacharias, N., Urban, S. E., Zacharias, M. I., et al. 2004, AJ, 127, 3043 